\title{
CRESCIMENTO DE MUDAS DE Sesbania virgata (Cav.) Pers. PLANTADAS EM UMA ÁREA DEGRADADA POR EXTRAÇÃO DE ARGILA
}

\author{
Marcos P. Coutinho*, José Geraldo de Araújo Carneiro**, Débora Guerra Barroso***, \\ Luciana Aparecida Rodrigues****, Fábio A. M. M. A. Figueiredo*****, \\ Andréa Vita R. Mendonça******, Adalberto Brito de Novaes******* \\ * Eng. Florestal, M.Sc., Centro de Ciências e Tecnologias Agropecuárias, UENF - mpzcoutinho@yahoo.com.br \\ ** Eng. Florestal, Dr., Centro de Ciências e Tecnologias Agropecuárias, UENF - carneiro@uenf.br \\ *** Eng ${ }^{\mathrm{a}}$. Agrônoma, Dr ${ }^{\mathrm{a}}$., Centro de Ciências e Tecnologias Agropecuárias, UENF - deborah@uenf.br \\ **** Eng ${ }^{\mathrm{a}}$. Florestal, Dr ${ }^{\mathrm{a}}$., Centro de Ciências e Tecnologias Agropecuárias, UENF - lua@uenf.br \\ ***** Mestrando em Produção Vegetal, UENF - fabio@yahoo.com.br \\ ****** Doutoranda em Produção Vegetal, UENF - andreavita.mendonça@bol.com.br \\ *******Eng. Agrônomo, Dr., Escola de Florestas, UESB - adalbert@uesb.br
}

Recebido para publicação: 28/11/2004 - Aceito para publicação: 31/08/2005

\begin{abstract}
Resumo
Crescimento de mudas de Sesbania virgata (Cav.) Pers. plantadas em uma área degradada por extração de argila. O objetivo deste trabalho foi testar o crescimento inicial de mudas de Sesbania virgata (Cav.) Pers. (sesbânia), plantadas em uma área degradada por extração de argila, em covas fertilizadas. As mudas foram inoculadas com rizóbio e fungos micorrízicos arbusculares. $\mathrm{O}$ experimento foi instalado em blocos ao acaso, com 4 repetições, sendo as parcelas constituídas por 16 plantas. Os tratamentos foram: 1 . testemunha; 2 . "ferkal" $\left(0,6 \mathrm{~kg} \mathrm{cova}^{-1}\right) ; 3$. composto de lixo urbano $\left(0,8 \mathrm{~kg} \mathrm{cova}^{-1}\right) ; 4$. torta de filtro $\left(0,6 \mathrm{~kg} \mathrm{cova}^{-1}\right)$; e 5 . superfosfato simples $\left(0,1 \mathrm{~kg} \mathrm{cova}^{-1}\right)$. Nos 3 primeiros meses, foram avaliados os percentuais de sobrevivência e, bimestralmente, o crescimento em altura e diâmetro ao nível do solo, até o $10^{\circ}$ mês após o plantio. Nessa ocasião, também foram determinados os teores foliares de $\mathrm{N}, \mathrm{P}$ e $\mathrm{K}$ e a produção de fitomassa, não tendo sido constatada diferença significativa entre os tratamentos para essas variáveis. Não foi verificado efeito da fertilização sobre o crescimento e a produção de fitomassa de sesbânia, nas condições deste estudo. $O$ comprimento e área superficial de raízes finas (diâmetros maiores e menores que $0,3 \mathrm{~mm}$ ) foram avaliados aos 14 meses, tendo-se constatado diferenças significativas. Verificou-se maior comprimento e superfície de raízes mais finas em comparação àquelas maiores que $0,3 \mathrm{~mm}$ de diâmetro. O composto de lixo urbano e a torta de filtro estimularam a produção de raízes.

Palavras-chave: Revegetação; fertilização; leguminosa; composto de lixo urbano; crescimento de raízes.
\end{abstract}

\begin{abstract}
Sesbania virgata (Cav.) Pers. seedlings growth in a degraded area after clay extraction. This experiment intended to evaluate the initial growth of Sesbania virgata (Cav.) Pers. (sesbânia) seedlings planted with distinct fertilizers in a degraded area after clay extraction. The seedlings were inoculated with Rhizobium and arbuscular mycorrhizal fungi. The experiment was carried out in a randomized complete-block design. Each experimental unit had 4 repetitions with 16 seedlings. The treatments were: 1 . control; 2. "ferkal" $\left(0,6 \mathrm{~kg} \mathrm{hole}^{-1}\right) ; 3$. urban waste $\left(0,8 \mathrm{~kg} \mathrm{hole}^{-1}\right) ; 4$. sugar cane filter cake $\left(0,6 \mathrm{~kg} \mathrm{hole}^{-1}\right)$ and 5 . simple super phosphate $\left(0,1 \mathrm{~kg} \mathrm{hole}^{-1}\right)$. Along the first 3 months the rate of surviving plants was evaluated. Height and diameter at the soil level had been bimonthly measured until the tenth month of growth. At this time were also measured the N, P and K nutrient contents of the leaves as well as the biomass production. No difference among the treatments had been observed for these variables. Effects of fertilization on the growth and production of biomass were not found out under the conditions of this study. Lenght and superficial thin root area (diameter larger and smaller than $0,3 \mathrm{~mm}$ ) were evaluated 14 months after planting. Higher values of length and thinner superficial area of the smallest roots were verified in comparison to the larger ones (more than $0,3 \mathrm{~mm}$ ). Urban waste and sugar cane filter cake stimulated root production.

Keywords: Reforestation; fertilization; vegetables; urban waste; root growth.
\end{abstract}




\section{INTRODUÇÃO}

No território brasileiro, estima-se a ocorrência de mais de 200 milhões de hectares de áreas degradadas, seja por ações naturais ou antrópicas. São originadas, principalmente da agricultura migratória, de pastagens não-sustentáveis, de ocupação imprópria de regiões urbanas, de enchentes e de assoreamentos dos rios. Áreas desmatadas e destinadas à agricultura, somadas às de pastagens, representam cerca de $69 \%$ das áreas degradadas no mundo, enquanto que áreas de mineração representam, aproximadamente, $1,2 \%$ (Cabral et al., 2002).

Apesar do baixo percentual que representa, a mineração é uma das atividades humanas que mais contribui para alteração da superfície terrestre, afetando o local explorado e seu entorno e provocando impactos sobre a água, o ar, o solo, o subsolo e a paisagem (Griffith, 1980; Sirtoli et al., 2002), visto que retira a camada orgânica do solo, porção onde se encontra a maior parte dos nutrientes disponíveis para as plantas (Kiehl, 1985), resultando em ambiente com baixa resiliência (Carpanezzi, Costa e Castro, 1990). Contudo, essa atividade é indispensável para atender à demanda da sociedade moderna, dada a importância assumida pelos bens minerais em praticamente todas as atividades humanas, desde as mais básicas, como habitação, saneamento básico, transporte e agricultura, até as mais sofisticadas, como tecnologia de ponta nas áreas de comunicação e medicina (Banco do Nordeste, 1999).

A matéria orgânica melhora as características físicas, químicas e favorece os microrganismos do solo, além de propiciar nutrientes para as plantas, que irão revegetar solos degradados (Santana Filho, Cardoso e Pereira, 1997; Pralon, 1999; Pralon e Martins, 2001).

Antes da revegetação, é fundamental minimizar os efeitos da topografia sobre as áreas degradadas, tendo, como objetivo, favorecer o restabelecimento, em menor período de tempo, da cobertura vegetal. As atividades com vistas à revegetação requerem seleção adequada de espécies para plantio e técnicas de manejo, visando à aceleração e à reconstituição dos processos naturais de sucessão (Oliveira e Ribeiro Jr., 2000; Dutra et al., 2002).

Pesquisas reportadas por Franco et al. (1994) e Nau e Sevegnani (1997) sugerem a utilização de espécies de rápido crescimento, que sejam capazes de gerar aporte de $\mathrm{N} \mathrm{e} \mathrm{C}$ ao solo e aumentar a disponibilidade dos demais nutrientes, melhorando o solo pela deposição de matéria orgânica e reciclagem de nutrientes. Essa técnica favorece o estabelecimento do processo sucessional, merecendo destaque as espécies da família Leguminosae (Piagentini et al., 2002).

Segundo Samôr (1999), a Sesbania virgata (Cav.) Pers. (sesbânia) é uma leguminosa pioneira, potencial para a revegetação de cavas de extração de argila, pela freqüência de ocorrência em cavas abandonadas e disponibilidade de sementes. De acordo com Santos, Moreira e Siqueira (1998), a sesbânia, também pode formar simbiose radicular com rizóbio e fungos micorrízicos arbusculares (FMA's), que ajudam na absorção de nutrientes em solos de baixa fertilidade.

São importantes as ações que determinam as técnicas mais adequadas para potencializar o estabelecimento dessa espécie nas cavas de extração de argila, acelerando o crescimento e o aporte de fitomassa, em curto espaço de tempo, promovendo melhorias no solo e o futuro estabelecimento de outras espécies.

Este experimento objetivou avaliar o estabelecimento e crescimento inicial de mudas de sesbânia, inoculadas com FMA e rizóbio, em cavas de extração de argila, fertilizadas nas covas, com diferentes subprodutos orgânicos e com superfosfato simples.

\section{MATERIAL E MÉTODOS}

Mudas de sesbânia foram produzidas, a partir de sementes, na Estação Experimental da PESAGRO, no município de Campos dos Goytacazes (RJ), cujas coordenadas geográficas são de $21^{\circ} 48^{\prime}$ $\mathrm{S}$ de latitude, $41^{\circ} 20^{\prime} \mathrm{W}$ de longitude e $11 \mathrm{~m}$ de altitude.

O substrato utilizado na produção das mudas foi constituído de bagaço de cana-de-açúcar e torta de filtro de usina açucareira (3:2, v:v), relação estudada por Morgado (1998). A torta de filtro é um subproduto, resíduo da fabricação de açúcar e álcool. Essa mistura, após estabilização, foi peneirada em tela de $0,4 \mathrm{~cm}$ e as partículas maiores foram trituradas em máquina desintegradora, sendo, posteriormente, misturadas ao material peneirado. Em seguida, adicionou-se inóculo de fungos micorrízicos arbusculares de cavas de extração de argila da região, na camada superior do substrato, que foi umedecido e prensado em blocos, conforme metodologia descrita nos trabalhos de Leles (1998) e Barroso (1999). 
As sementes de sesbânia tiveram sua dormência tegumentar superada em água quente, até retorno à temperatura ambiente, conforme recomendado por Franco et al. (1992). A seguir, foi adicionado rizóbio específico para sesbânia, o isolado (BR 5401 e BR 5412), fornecido pela EMBRAPA/CNPAB, Seropédica (RJ). A semeadura foi feita manualmente, colocando-se duas sementes em cada um dos 40 pontos de semeadura dos blocos. Uma semana após a emergência, deixou-se a plântula mais vigorosa por ponto de semeadura.

\section{Caracterização da área do plantio}

O plantio foi realizado em uma cava de extração de argila localizada no distrito de Campo Limpo ( $21^{\circ} 51^{\prime} \mathrm{S}$ de latitude, $41^{\circ} 14^{\prime} \mathrm{W}$ de longitude e $14 \mathrm{~m}$ de altitude), pertencente à Cerâmica Stilbe Ltda., em Campos dos Goytacazes (RJ), apresentando uma profundidade média de $2 \mathrm{~m}$. A camada superficial, retirada antes da extração de argila, foi acumulada em murunduns na própria cava. Logo após a extração, o material retirado e as sobras de entulhos das cerâmicas foram espalhados e aplainados por meio de gradagem. A espessura da camada distribuída não foi regular, apresentando profundidades que oscilaram entre 10 e $20 \mathrm{~cm}$. Esse preparo consiste no procedimento adotado pelos ceramistas após a extração de argila.

\section{Fertilização e plantio}

Os tratamentos, constituídos pela aplicação de diferentes fertilizantes, nas covas de plantio, foram: 1. testemunha; 2. "ferkal" $\left(0,6 \mathrm{~kg} \mathrm{cova}^{-1}\right) ; 3$. composto de lixo urbano $\left(0,8 \mathrm{~kg}\right.$ cova $\left.{ }^{-1}\right) ; 4$. torta de filtro de usina açucareira $\left(0,6 \mathrm{~kg} \mathrm{cova}^{-1}\right)$; e 5 . superfosfato simples $\left(0,1 \mathrm{~kg} \mathrm{cova}^{-1}\right)$, valores apresentados em base seca.

Para o cálculo da base seca, os subprodutos foram colocados em estufa de circulação forçada de ar, a $70{ }^{\circ} \mathrm{C}$, por um período de 48 horas, quando as amostras atingiram peso constante. Nesse período, os percentuais de umidade foram 70,50 e $0 \%$, respectivamente, para torta de filtro, "ferkal" e composto de lixo urbano.

O "ferkal", é um subproduto resultante da fabricação de ácido lático, e o lixo urbano foi produzido pela usina de triagem e compostagem do município de Quissamã (RJ).

Com a utilização de um sulcador, abriram-se sulcos de $20 \mathrm{~cm}$ de profundidade a cada 3 metros, com auxílio do trator agrícola, nos quais foram executados o coveamento e plantio. $\mathrm{O}$ espaçamento

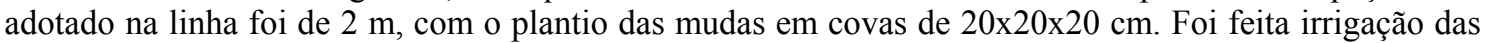
mudas após o plantio, sendo adicionados cerca de 2 L de água por cova, uma vez por semana, durante um mês.

O experimento foi instalado em blocos ao acaso, com 5 tratamentos e 4 repetições, com as parcelas constituídas por 16 mudas. Antes do plantio, foi feita caracterização química (profundidade de 0-30 cm) e microbiológica (profundidade de $0-10 \mathrm{~cm}$ ) do substrato da cava, ambas compostas por 4 amostras por bloco (Tabela 1).

Os microrganismos foram contados através do método de diluição em série, com 4 repetições, utilizando-se, para bactérias, o meio de Bunt e Rovira (1995), pH 7,4, adicionado de actidione. A contagem dos fungos foi realizada após sua multiplicação em meio de Martin (1950), pH 5,5, acrescido de $70 \mathrm{mg} \mathrm{L}^{-1}$ de rosa-de-bengala e $100 \mathrm{mg} \mathrm{L}^{-1}$ de uma mistura de penicilina e estreptomicina. $\mathrm{O}$ número de microrganismos solubilizadores de fosfato foi determinado através da contagem em placas de Petri, de acordo com procedimentos adotados por Nahas, Ceturion e Assis (1994). Os subprodutos também foram caracterizados quimicamente (Tabela 2).

\section{Avaliações}

As plantas foram monitoradas quanto à sobrevivência, nos três primeiros meses, e bimestralmente, quanto à altura e diâmetro ao nível do solo, até o décimo mês após o plantio.

Dez meses após o plantio, determinaram-se os teores foliares de $\mathrm{N}$ (Nessler, após digestão sulfúrica), P (colorimetria) e K (espectrofotometria de emissão de chama), segundo Jones Jr., Wolf e Mills (1991) e Malavolta, Vitti e Oliveira (1997), sendo tomadas amostras de uma planta por parcela. Nessa época, foi realizada poda das plantas, mantendo-se apenas o ramo principal, e o material depositado sobre o solo foi quantificado. Foi realizada a pesagem do material podado de duas plantas por parcela, englobando folhas, galhos e frutos, para quantificação da fitomassa a ser incorporada com essa prática. O material vegetal proveniente da poda da sesbânia foi deixado sobre o solo para ser decomposto naturalmente. 
Tabela 1. Caracterização química e microbiológica da área do experimento, na ocasião do plantio de mudas de Sesbania virgata (Cav.) Pers.

Table 1. Chemical e microbiological characterization of experimental area, during sesbânia seedlings planting.

\begin{tabular}{|c|c|}
\hline Características & Valores \\
\hline $\mathrm{pH}$ em $\mathrm{H}_{2} \mathrm{O}$ & 5,6 \\
\hline $\mathrm{P}^{*}\left(\mathrm{mg} \cdot \mathrm{dm}^{-3}\right)$ & 18,4 \\
\hline $\mathrm{K}^{*}\left(\mathrm{mg} \cdot \mathrm{dm}^{-3}\right)$ & 35,6 \\
\hline $\mathrm{Ca}\left(\mathrm{cmol}_{\mathrm{c}} \mathrm{dm}^{-3}\right)$ & 8,2 \\
\hline $\mathrm{Mg}\left(\mathrm{cmol}_{\mathrm{c}} \mathrm{dm}^{-3}\right)$ & 4,1 \\
\hline $\mathrm{Al}\left(\mathrm{cmol}_{\mathrm{c}} \mathrm{dm}^{-3}\right)$ & 0,0 \\
\hline $\mathrm{H}+\mathrm{Al}\left(\mathrm{cmol}_{\mathrm{c}} \mathrm{dm}^{-3}\right)$ & 3,6 \\
\hline $\mathrm{Na}\left(\mathrm{cmol}_{\mathrm{c}} \mathrm{dm}^{-3}\right)$ & 1,71 \\
\hline $\mathrm{C}(\%)$ & 1,56 \\
\hline $\mathrm{MO}\left(\mathrm{g} \mathrm{dm}^{-3}\right)$ & 26,9 \\
\hline $\mathrm{V}(\%)$ & 80 \\
\hline $\mathrm{Fe}\left(\mathrm{mg} \mathrm{dm}^{-3}\right)$ & 58,0 \\
\hline $\mathrm{Cu}\left(\mathrm{mg} \mathrm{dm}^{-3}\right)$ & 2,1 \\
\hline $\mathrm{Zn}\left(\mathrm{mg} \mathrm{dm}^{-3}\right)$ & 5,4 \\
\hline $\mathrm{Mn}\left(\mathrm{mg} \mathrm{dm}^{-3}\right)$ & 19,8 \\
\hline \multicolumn{2}{|l|}{ Microrganismos } \\
\hline Fungo total (mic. g solo ${ }^{-1}$ ) & 600 \\
\hline Bactérias solubilizadoras de fosfato (mic. $\mathrm{g} \mathrm{solo}^{-1}$ ) & $4,7 \times 10^{4}$ \\
\hline Bactérias total (mic. g solo ${ }^{-1}$ ) & $10,25 \times 10^{4}$ \\
\hline
\end{tabular}

Tabela 2. Análise química dos subprodutos utilizados na fertilização das covas para o plantio de mudas de Sesbania virgata (Cav.) Pers.

Table 2. Chemical analysis of by-products used to fertilization of holes for sesbania seedlings planting.

\begin{tabular}{|c|c|c|c|}
\hline \multirow{2}{*}{ Características } & \multicolumn{3}{|c|}{ Subprodutos } \\
\hline & Ferkal & Composto de lixo & Torta de filtro \\
\hline $\mathrm{pH} \mathrm{em} \mathrm{H}_{2} \mathrm{O}$ & 11,0 & 8,2 & 7,2 \\
\hline $\mathrm{P} *\left(\mathrm{mg}^{2} \mathrm{dm}^{-3}\right)$ & 22,0 & 1025,0 & 2460,0 \\
\hline $\mathrm{K}^{*}\left(\mathrm{mg} \cdot \mathrm{dm}^{-3}\right)$ & 36,0 & 1189,0 & 258,0 \\
\hline $\mathrm{Ca}\left(\mathrm{cmol}_{\mathrm{c}} \mathrm{dm}^{-3}\right)$ & 196,8 & 9,7 & 8,5 \\
\hline $\mathrm{Mg}\left(\mathrm{cmol}_{\mathrm{c}} \mathrm{dm}^{-3}\right)$ & 0,1 & 2,3 & 2,1 \\
\hline $\mathrm{Al}\left(\mathrm{cmol}_{\mathrm{c}} \mathrm{dm}^{-3}\right)$ & 0,0 & 0,0 & 0,0 \\
\hline $\mathrm{H}+\mathrm{Al}\left(\mathrm{cmol}_{\mathrm{c}} \mathrm{dm}^{-3}\right)$ & 0,0 & 0,0 & 0,0 \\
\hline $\mathrm{Na}\left(\mathrm{cmol}_{\mathrm{c}} \mathrm{dm}^{-3}\right)$ & 0,11 & 1,74 & 0,03 \\
\hline C (\%) & 2,47 & 2,76 & 5,1 \\
\hline $\mathrm{MO}\left(\mathrm{g} \mathrm{dm}^{-3}\right)$ & 42,6 & 47,6 & 87,6 \\
\hline V (\%) & 100 & 100 & 97 \\
\hline $\mathrm{Fe}\left(\mathrm{mg} \mathrm{dm}^{-3}\right)$ & 3,0 & 78,0 & 153,0 \\
\hline $\mathrm{Cu}\left(\mathrm{mg} \mathrm{dm}^{-3}\right)$ & 0,1 & 4,2 & 6,7 \\
\hline $\mathrm{Zn}\left(\mathrm{mg} \mathrm{dm}^{-3}\right)$ & 0,1 & 33,0 & 20,0 \\
\hline $\operatorname{Mn}\left(\mathrm{mg} \mathrm{dm}^{-3}\right)$ & 6,2 & 48,3 & 79,8 \\
\hline
\end{tabular}


Quatorze meses após o plantio, quatro meses após a poda da parte aérea, foram retiradas amostras de solo nos $17 \mathrm{~cm}$ superficiais, com trado retangular, em uma planta de cada parcela, a distâncias de 30 e $60 \mathrm{~cm}$ do colo das plantas, para quantificação de raízes. As medições foram efetuadas por meio do programa QuantRoot desenvolvido no Departamento de Solos da UFV, o qual realiza medição das raízes em imagens digitalizadas em escala real, gerando o programa linhas de igual comprimento ao das raízes.

Os dados foram submetidos à análise de variância e as diferenças comparadas por testes de Tukey, ao nível de 5\% de probabilidade.

\section{RESULTADOS E DISCUSSÃO}

As características químicas, a disponibilidade de $\mathrm{P}, \mathrm{K}$ e de matéria orgânica do substrato (Tabela 1) e dos subprodutos (Tabela 2), permitiram condições de crescimento das mudas de sesbânia após plantio em cava de extração de argila (Figura 1).

Não se constatou diferença entre os tratamentos, com relação ao percentual de sobrevivência (100\% em todas as parcelas), crescimento em altura e em diâmetro, durante os 10 meses de avaliação. A Figura 1 ilustra o crescimento médio dos tratamentos, considerando-se não ter sido detectada diferença significativa entre eles.

Pelo acompanhamento inicial da sobrevivência e do crescimento das plantas de sesbânia, constatou-se que a espécie adaptou-se ao local de plantio sem necessidade de uso dos fertilizantes e das quantidades em estudo. A altura das plantas passou de $0,40 \mathrm{~m}$ para $3 \mathrm{~m}$ em 10 meses, indicando a rapidez de crescimento dessa espécie no campo, o que é de grande importância em plantio em áreas degradadas.

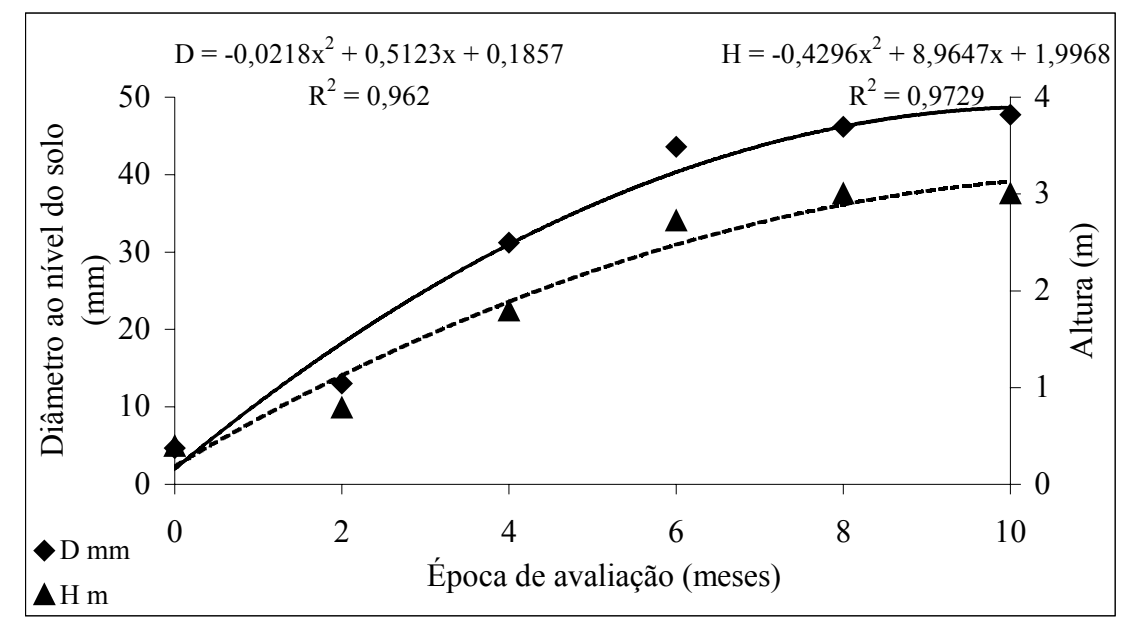

Figura 1. Crescimento em altura e diâmetro ao nível do solo, até 10 meses após o plantio das mudas de sesbânia em cavas de extração de argila.

Figure 1. Height and ground level diameter growth until 10 months after planting sesbania seedlings in a clay extraction area.

O crescimento em altura tendeu a estabilizar-se do $8^{\circ}$ para o $10^{\circ}$ mês (Figura 1), assim como as envergaduras das copas que, já aos 8 meses, tocavam-se entre as linhas de plantio. Embora não tenha havido diferença significativa entre os tratamentos, cada planta de sesbânia produziu, após a poda, em média, 4,86 kg de fitomassa fresca. O rápido crescimento da parte aérea é importante em trabalhos de recuperação de áreas degradadas (Franco et al., 1994; Nau e Sevegnani, 1997; Faria, Davide e Botelho, 1997; Faria et al., 2002), uma vez que pode cobrir o solo e produzir grande quantidade de folhas. A queda das folhas no solo é importante para a ciclagem de nutrientes e o acréscimo da matéria orgânica de baixa relação $\mathrm{C} / \mathrm{N}$, favorecendo as características biológicas, físicas e químicas do solo. 
As plantas de sesbânia apresentavam em média os seguintes teores foliares: $37,49 \mathrm{~g} \mathrm{~kg}^{-1} \mathrm{de} \mathrm{N}$, $2,90 \mathrm{~g} \mathrm{~kg}^{-1}$ de P e 11,66 $\mathrm{g} \mathrm{kg}^{-1}$ de K. Rodrigues (2001) encontrou teor foliar de P semelhante estudando a mesma espécie, com mudas inoculadas com fungos micorrízicos arbusculares (FMA's) com e sem rizóbio. As plantas inoculadas com rizóbio e rizóbio mais FMA's apresentaram teor foliar de $\mathrm{N}$ de aproximadamente $27 \mathrm{~g} \mathrm{~kg}^{-1}$. Todos os tratamentos foram inferiores ao do presente estudo, o que indica boa fixação biológica de $\mathrm{N}$ e que a sesbânia supre suas demandas nutricionais e é capaz de desenvolver-se nesse tipo de substrato independentemente da adubação utilizada. Pode-se inferir que a prática adotada de recobrir a cava com a camada orgânica, assim como o regime pluviométrico favorável, ocorrido após o plantio, tenham exercido relevante papel no crescimento inicial das mudas.

Santos (2000) encontrou efeito negativo da adição de doses de P ao solo, analisando os teores foliares de $\mathrm{N}$ em sesbânia. Sem a adição de $\mathrm{P}$, todos os tratamentos apresentaram teores foliares maiores que $40 \mathrm{~g} \mathrm{~kg}^{-1}$. O teor de $\mathrm{N}$, presente nas folhas de sesbânia nos diferentes trabalhos, mostra a capacidade da espécie em fixar esse nutriente.

A incorporação da fitomassa produzida pela sesbânia pode aumentar o $\mathrm{N}$ e outros nutrientes do solo, minimizando os efeitos que a baixa disponibilidade de nutrientes causa no processo sucessional e produtivo. Esse material, incorporado ao solo, poderá também favorecer o crescimento de outras espécies a serem introduzidas, ou que surjam espontaneamente. A melhoria do solo por plantas leguminosas, conforme Griffith, Dias e Marco Jr. (2000), possibilita menor intensidade de intervenção futura no sistema e, devido ao melhor condicionamento do substrato, facilita o estabelecimento de outras plantas na seqüência do processo sucessional.

Faria et al. (2002), estudando espécies arbóreas e arbustivas, leguminosas ou não, na revegetação de talude de estéril de mineração de ferro, encontraram grande variação no crescimento das espécies introduzidas na área. Sesbania exasperta foi a espécie que apresentou maior crescimento durante a avaliação, embora sua produção de biomassa tenha sido menor do que a observada para Mimosa artemisiana.

Para Gonçalves-Alvim et al. (2002), a utilização de leguminosas na revegetação de áreas degradadas é conhecida pela eficiência na fixação de $\mathrm{N}_{2}$, que, em geral, é muito pouco disponível ou ausente em áreas de mineração de superfície. Além disso, raízes de leguminosas contribuem para melhorar a agregação do solo nessas áreas, facilitando a colonização por outras espécies.

$\mathrm{Na}$ Tabela 3 são observados os valores de comprimento e área superficial de raízes finas, amostradas da camada superficial do solo, a uma profundidade de $17 \mathrm{~cm}$, e com valores maiores e menores de 0,3 mm, coletadas a 30 e $60 \mathrm{~cm}$ do tronco das plantas de sesbânia, aos 14 meses após o plantio.

Pode-se observar (Tabela 3) que, de maneira geral, houve uma proliferação na produção de raízes mais finas, constatando-se cerca de $446 \%$ a mais na soma dos comprimentos dessas raízes em confronto com as raízes maiores que $0,3 \mathrm{~mm}$. Por outro lado, a área superficial de raízes finas foi $168 \%$ maior quando comparada à das raízes com diâmetro superior a $0,3 \mathrm{~mm}$. Essa maior produção de raízes finas pode justificar a capacidade da sesbânia em apresentar alto teor de nutrientes nas folhas independentemente da adubação testada.

As plantas que foram fertilizadas com composto de lixo urbano apresentaram maior produção de raízes finas com diâmetro acima de $0,3 \mathrm{~mm}$ (média de $0,41 \mathrm{~mm}$ ), com relação aos demais tratamentos, enquanto que as plantas fertilizadas com torta de filtro produziram maior quantidade de raízes com diâmetro abaixo de $0,3 \mathrm{~mm}$ que os demais tratamentos. Essas diferenças foram acentuadas nas amostras tomadas a $30 \mathrm{~cm}$ do tronco, mostrando o efeito desses subprodutos no estímulo ao enraizamento.

As raízes finas são importantes no acesso aos recursos do solo, podendo ser indicadores da capacidade de absorção de nutrientes (Rylter, 1997). Essas raízes são importantes na incorporação de matéria orgânica ao solo, concentrando-se de 40 a $70 \%$ no horizonte orgânico. As raízes mortas podem representar 50-80\% dessa biomassa (Vogt, Grier e And Vogt, 1986).

Os trabalhos com leguminosas têm mostrado que essas espécies são indicadas para realizar rápido recobrimento do solo. $\mathrm{O}$ presente estudo comprovou a capacidade da sesbânia em produzir, com rapidez, grande quantidade de fitomassa para o recobrimento do substrato das cavas de extração de argila, independentemente da adição de adubos químicos ou orgânicos. Entretanto, observa-se que tanto o lixo quanto a torta de filtro estimularam a produção de raízes finas, favorecendo o incremento de matéria orgânica e, conseqüentemente, melhorando as condições para introdução de outras espécies. Infere-se, portanto, que essa capacidade de estímulo possa contribuir para futura diferenciação de crescimento entre covas fertilizadas ou não. 
Tabela 3. Comprimento e área superficial de raízes finas de Sesbania virgata (Cav.) Pers., amostradas da camada superficial do solo (profundidade de $17 \mathrm{~cm}$ ), aos 14 meses após o plantio em covas fertilizadas.

Table 3. Length and superficial area of sesbânia thin roots, collected from the soil surface (depth of 17 $\mathrm{cm}) 14$ months after planting in fertilized holes.

\begin{tabular}{|c|c|c|c|c|}
\hline \multirow{3}{*}{ Fertilização } & \multicolumn{4}{|c|}{ Comprimento de raízes finas $(\mathrm{cm})$ por volume de solo $\left(\mathrm{cm}^{-3}\right)$} \\
\hline & \multicolumn{2}{|c|}{ Diâmetro $>0,3$ mm } & \multicolumn{2}{|c|}{ Diâmetro $<0,3$ mm } \\
\hline & $30 \mathrm{~cm}$ da planta & $60 \mathrm{~cm}$ da planta & $30 \mathrm{~cm}$ da planta & $60 \mathrm{~cm}$ da planta \\
\hline Testemunha & $0,090 \mathrm{~b} \mathrm{~A}$ & 0,099 a $\mathrm{A}$ & $0,897 \mathrm{~b} \mathrm{~A}$ & 0,823 a $\mathrm{A}$ \\
\hline Ferkal & $0,144 \mathrm{ab} \mathrm{A}$ & 0,118 a $\mathrm{A}$ & 0,752 bc A & 0,791 a $\mathrm{A}$ \\
\hline Lixo & 0,363 a $\mathrm{A}$ & 0,104 a B & 0,613 с A & 0,760 a $\mathrm{A}$ \\
\hline Torta & $0,152 \mathrm{ab} \mathrm{A}$ & 0,129 a $\mathrm{A}$ & 1,672 a A & $0,479 \mathrm{~b} \mathrm{~B}$ \\
\hline Superfosfato & $0,098 \mathrm{~b} \mathrm{~A}$ & 0,105 a $\mathrm{A}$ & $0,520 \mathrm{c} \mathrm{A}$ & $0,348 \mathrm{~b} \mathrm{~A}$ \\
\hline Total & \multicolumn{2}{|c|}{0,140} & \multicolumn{2}{|c|}{0,765} \\
\hline
\end{tabular}

\begin{tabular}{|c|c|c|c|c|}
\hline \multirow{3}{*}{ Fertilização } & \multicolumn{4}{|c|}{ Área superficial de raízes finas $\left(\mathrm{cm}^{2}\right)$ por volume de solo $\left(\mathrm{cm}^{-3}\right)$} \\
\hline & \multicolumn{2}{|c|}{ Diâmetro $>0,3 \mathrm{~mm}$} & \multicolumn{2}{|c|}{ Diâmetro $<0,3 \mathrm{~mm}$} \\
\hline & $30 \mathrm{~cm}$ da planta & $60 \mathrm{~cm}$ da planta & $30 \mathrm{~cm}$ da planta & $60 \mathrm{~cm}$ da planta \\
\hline Testemunha & $0,013 \mathrm{~b} \mathrm{~A}$ & 0,013 a A & $0,061 \mathrm{~b} \mathrm{~A}$ & $0,051 \mathrm{a} \mathrm{A}$ \\
\hline Ferkal & $0,019 \mathrm{~b} \mathrm{~A}$ & 0,015 a $\mathrm{A}$ & 0,052 bc $\mathrm{A}$ & $0,054 \mathrm{a} \mathrm{A}$ \\
\hline$\overline{\text { Lixo }}$ & 0,052 a $\mathrm{A}$ & 0,013 a B & 0,041 bc $\mathrm{A}$ & 0,048 a $\mathrm{A}$ \\
\hline Torta & $0,021 \mathrm{~b} \mathrm{~A}$ & 0,016 a A & 0,116 a A & $0,033 \mathrm{ab} \mathrm{B}$ \\
\hline Ad. química & $0,015 \mathrm{~b} \mathrm{~A}$ & 0,015 a $A$ & $0,035 \mathrm{c} \mathrm{A}$ & $0,023 \mathrm{~b} \mathrm{~A}$ \\
\hline Total & \multicolumn{2}{|c|}{0,019} & \multicolumn{2}{|c|}{0,051} \\
\hline
\end{tabular}

Médias seguidas pela mesma letra minúscula na coluna e maiúscula na linha, dentro de cada faixa de diâmetro, não diferem entre si pelo teste de Tukey, ao nível de $5 \%$ de probabilidade.

\section{CONCLUSÕES}

- A sesbânia pode ser indicada para revegetação de cavas de extração de argila, com potencial de rápida incorporação de fitomassa ao sistema.

- Os subprodutos não influenciam o crescimento das mudas dessa espécie, nos 10 primeiros meses após o plantio, nas condições de solo deste estudo.

- A adição nas covas de composto de lixo urbano e a torta de filtro estimulam a produção de raízes finas nas plantas da espécie estudada.

\section{AGRADECIMENTOS}

Os autores expressam seus agradecimentos à FENORTE, UENF e a FAPERJ, pelo apoio financeiro, e à Cerâmica Stilbe Ltda., pela cessão da área e pelo auxílio prestado.

\section{REFERÊNCIAS}

BANCO DO NORDESTE (Brasil). Manual de impactos ambientais: orientações básicas sobre aspectos ambientais de atividades produtivas. In: Dias, M. do C. O. (Coord.), Pereira, M. C. B, Dias, P. L. F, Virgílio, J. F. Fortaleza, CE: Banco do Nordeste., 1999, 297 p.

BARROSO, D. G. Qualidade de mudas de Eucalyptus camaldulensis e Eucalyptus urophylla, produzidas em tubetes e em blocos prensados, com diferentes substratos. Campos dos Goytacazes, RJ, 1999. 83 p. Tese (Doutorado em Produção Vegetal) - Universidade Estadual do Norte Fluminense.

BUNT, J. S.; ROVIRA, A. D. Microbiological studies of somes subantartic soils. The Journal of Soil Science, Oxford, v.6, p.119-128, 1995. 
CABRAL, V. M.; FARIA, S. M. de; DIAS, G. B. N.; LOTT, C. M.; NARA, H. C. Seleção de espécies leguminosas fixadoras de nitrogênio para utilização na recuperação de áreas mineradas pela Companhia vale do Rio Doce. In: SIMPÓSIO NACIONAL SOBRE RECUPERAÇÃO DE ÁREAS DEGRADADAS "ÁGUA E BIODIVERSIDADE", 5., 2002, Belo Horizonte, MG. Anais... Belo Horizonte: SOBRADE, 2002. p.463-465.

CARPANEZZI, A. A.; COSTA, L. G. L.; CASTRO, C. Espécies pioneiras para a recuperação de áreas degradadas: a observação de laboratórios naturais. In: CONGRESSO FLORESTAL BRASILEIRO, 6. Campos de Jordão, SP, 1990. Anais...Campos de Jordão: BS/SBEF, 1990, p.216-221.

DUTRA, G. C.; BOTELHO, S. A.; FERREIRA, C. A. G.; DAVIDE, A. C. Avaliação do crescimento de espécies arbóreas plantadas em duas estratégias de recuperação de áreas degradadas pela mineração. In: SIMPÓSIO NACIONAL SOBRE RECUPERAÇÃO DE ÁREAS DEGRADADAS "ÁGUA E BIODIVERSIDADE", 5., 2002, Belo Horizonte, MG. Anais... Belo Horizonte: SOBRADE, 2002. p.331333.

FARIA, J. M. R.; DAVIDE, A. C.; BOTELHO, S. A. Comportamento de espécies florestais em área degradada, com duas adubações de plantio. Cerne, Lavras, MG, v.3, n.1, p.25-44, 1997.

FARIA, S. M. de.; SILVA, M. G.; GRAIG, J.; DIAS, S. J.; LIMA, H. C.; NARA, M. Revegetação com espécies arbóreas fixadoras de nitrogênio em taludes de exploração de ferro na Samarco Minerações Mariana, MG. In: SIMPÓSIO NACIONAL SOBRE RECUPERAÇÃO DE ÁREAS DEGRADADAS "ÁGUA E BIODIVERSIDADE", 5., 2002, Belo Horizonte, MG. Anais... Belo Horizonte: SOBRADE, 2002. p.521-522.

FRANCO, A. A.; CAMPELO, E. F.; SILVA, E. M. R.; FARIA, S. M. Revegetação de solos degradados. Seropédica, Comunicado Técnico, EMBRAPA-CNPAB, 1992, n.9, 9p.

FRANCO, A. A.; CAMPELlO, E. F.; DIAS, L. E.; FARIA, S. M. de. Revegetação de áreas de mineração em Porto Trombetas - PA com leguminosas arbóreas noduladas e micorrizadas. In: SIMPÓSIO SUL-AMERICANO E II SIMPÓSIO NACIONAL SOBRE RECUPERAÇÃO DE ÁREAS DEGRADADAS, 1., 1994, Foz do Iguaçu, PR. Anais... Curitiba: FUPEF, 1994. p. 145-153.

GONÇALVES-ALVIM, S. J.; ALMEIDA, C.; KRAFETUSKI, A. C., FERNANDES, F., CLETO, S. FERNANDES, G. W. Diversidade de plantas em áreas degradadas no quadrilátero ferrífero, Minas Gerais. In: SIMPÓSIO NACIONAL SOBRE RECUPERAÇÃO DE ÁREAS DEGRADADAS "ÁGUA E BIODIVERSIDADE", 5., 2002, Belo Horizonte, MG. Anais... Belo Horizonte: SOBRADE, 2002. p.152154.

GRIFFITH, J. J. Recuperação conservacionista da superfície de áreas mineradas: uma revisão de literatura. Boletim Técnico, Viçosa, MG, n.79, p.106, 1980.

GRIFFITH, J. J., DIAS, L. D. MARCO JR, P. de. A recuperação ambiental. Ação Ambiental, Viçosa, MG, n.10, fev./mar., , p.8-10, 2000.

JONES JR., J. B.; WOLF, B.; MILLS, H. A. Plant Analysis Handbook: a practical sampling, preparation, analysis and interpretation guide. Athens (USA): Micro-macro publishing, 1991, 213p.

KIEHL, E. J. Fertilizantes orgânicos. Piracicaba, Ceres, 1985, 492p.

LELES, P. S. S. Produção de mudas de Eucalyptus camaldulensis, Eucalyptus grandis e Eucalyptus pellita em blocos prensados e em tubetes. Campos dos Goytacazes, RJ, 1998. 73p. Tese (Doutorado em Produção Vegetal) - Universidade Estadual do Norte Fluminense.

MAlavolta, E.; VITTI, G. C.; OlIVEIRA, S. A. de. Avaliação do estado nutricional das plantas, princípios e aplicações. 2.ed. Piracicaba: Potafos, 1997. 319p.

MARTIN, J. P. Use of acid, rose Bengal, and streptomycin in the plate method for estimating soil fungi. Soil Science, Baltimore, v.69, p.215-232, 1950.

MORGADO, I. F. Resíduos agro-industriais prensados como substrato para a produção de mudas de Eucalyptus grandis Hill ex Maiden e Saccharum spp. Campos dos Goytacazes, RJ. Universidade Estadual do Norte Fluminense, 1998. 102p. Tese (Doutorado em Produção Vegetal). 
NAHAS, E.; CETURION, J. F.; ASSIS, L. C. Microrganismos solubilizadores de fosfato e produtores de fosfatases de vários solos. Revista Brasileira Ciência do Solo, Viçosa, MG, v.18, p.43-48, 1994.

NAU, S. R.; SEVEGNANI, L. Vegetação recolonizadora em mina de argila e propostas para recuperação ambiental. In: SIMPÓSIO NACIONALL SOBRE RECUPERAÇÃO DE ÁREAS DEGRADADAS, 3. 1997, Ouro Preto, MG, Anais... Ouro Preto: SOBRADE-SIF, 1997. p.54-66.

PRALON, A. Z. Produção de mudas de Mimosa caesalpiniaefolia, inoculadas com fungos micorrízicos arbusculares e rizóbio, em estéril de argila misturado com o resíduo Ferkal. Campos dos Goytacazes, RJ. 1999. 70p. Dissertação (Mestrado em Produção Vegetal) - Universidade Estadual do Norte Fluminense.

PRALON, A. Z.; MARTINS, M. A. Utilização do resíduo industrial ferkal na produção de mudas de Mimosa caesalpiniaefolia, em estéril de extração de argila, inoculadas com fungos micorrízicos arbusculares e rizóbio. Revista Brasileira Ciência do Solo, Viçosa, MG,. v.25, n.1, p.55-63, 2001.

OLIVEIRA, D. M.; RIBEIRO JR., E. S. Técnicas gerais aplicadas à recuperação de áreas degradadas pela atividade de mineração. Ação Ambiental, Viçosa, MG, n.10, fev./mar., p.16-18, 2000.

PIAGENTINI, P. M.; DIAS, L. E.; CAMPELLO, E. F. C.; RIBEIRO JR, E. S. Crescimento de diferentes espécies arbóreas e arbustivas em depósito de beneficiamento de minérios de zinco em Vazante, MG. In: SIMPÓSIO NACIONAL SOBRE RECUPERAÇÃO DE ÁREAS DEGRADADAS "ÁGUA E BIODIVERSIDADE", 5., Belo Horizonte, MG. Anais... Belo Horizonte: SOBRADE, 2002. p.413-415.

RODRIGUES, L. A. Crescimento e absorção de nutrientes por plantas de Eucalyptus grandis e leguminosas em resposta à inoculação com fungos micorrízicos arbusculares e rizóbio. Campos dos Goytacazes, RJ. 2001. 101p. Tese (Doutorado em Produção Vegetal) - Universidade Estadual do Norte Fluminense,

RYLTER, R. M. Fine root production and carbon and nitrogen allocation in basket willows. Upsalla, Suécia, 1997. Doctoral Thesis - Swedish University of Agricultural Sciences

SAMÔR, O. J. M. Comportamento de mudas de Sesbania virgata e Anadenanthera macrocarpa, produzidas em diferentes recipientes e substratos, destinadas à recuperação de áreas degradadas pela extração de argila. Campos dos Goytacazes, RJ,1999. 70p. Dissertação (Mestrado em Produção Vegetal) - Universidade Estadual do Norte Fluminense.

SANTANA FILHO, S.; CARDOSO, I. M.; PEREIRA, J. T. Utilização orgânica de lixo urbano na recuperação de áreas degradadas. In: SIMPÓSIO NACIONAL SOBRE RECUPERAÇÃO DE ÁREAS DEGRADADAS, 3., Ouro Preto, MG,1997. Anais...Ouro Preto: SOBRADE-SIF, 1997. p.194-204.

SANTOS, D. R. dos. Fósforo, fungo micorrízico e Azorhizobium na produção de fitomassa e nutrição de Sesbania virgata (Cav.) Per e Sesbania rostrada (Brem. \& Oberm.) Lavras: Universidade Federal de Lavras, MG, 2000. 88p. Dissertação (Mestrado em Agronomia/Solo e Nutrição de Planta) - UFLA.

SANTOS, D. R.; MOREIRA, F. M. S.; SIQUEIRA, J. O. Fósforo, fungos micorrízicos arbusculares e rizóbio no crescimento, modulação e fixação biológica de nitrogênio em Sesbania virgata e Sesbania rostrata. In: REUNIÃO BRASILEIRA DE FERTILIDADE DO SOLO E NUTRIÇÃO DE PLANTAS, REUNIÃO BRASILEIRA SOBRE MICORRIZAS, 7., SIMPÓSIO BRASILEIRO DE MICROBIOLOGIA DO SOLO, 5., REUNIÃO BRASILEIRA DE BIOLOGIA DO SOLO. 2., 23.,1998, Caxambu, MG. Anais... 1998, 204p.

SIRTOLI, A. E.; CRUZ, P. T. D.; SIRTOLI, A. R.dos A..; FERREIRA, F. V. Diagnóstico da recuperação ambiental em pequenas mineradoras de calcário na região metropolitana de Curitiba, PR.. In: SIMPÓSIO NACIONAL SOBRE RECUPERAÇÃO DE ÁREAS DEGRADADAS "ÁGUA E BIODIVERSIDADE", 5., 2002, Belo Horizonte, MG. Anais... Belo Horizonte: SOBRADE, 2002, p. 405-406.

VOGT, K. A.; GRIER, C. C.; VOGT, D. J. Production, turnover, and nutrient dynamics of above and belowground detritus of world forests. Advances in Ecological Research, n.15, p.303-377. 1986. 